Full-width low AC loss coated conductor production by substrate modification

This content has been downloaded from IOPscience. Please scroll down to see the full text. 2015 Supercond. Sci. Technol. 28090501

(http://iopscience.iop.org/0953-2048/28/9/090501)

View the table of contents for this issue, or go to the journal homepage for more

Download details:

IP Address: 131.111.184.102

This content was downloaded on 05/10/2015 at $16: 54$

Please note that terms and conditions apply. 


\section{Full-width low AC loss coated conductor production by substrate modification}

\author{
S C Hopkins \\ Department of Materials Science \\ and Metallurgy, University of \\ Cambridge, 27 Charles Babbage \\ Road, Cambridge CB3 OFS, UK \\ E-mail: sch29@cam.ac.uk
}

This is a viewpoint on the fast track communication by A C Wulff et al (2015 Supercond. Sci. Technol. 28 072001). Impressive progress continues to be made in improving the critical current performance of $(\mathrm{Y}, \mathrm{Gd}) \mathrm{Ba}_{2} \mathrm{Cu}_{3} \mathrm{O}_{7-\delta}(\mathrm{REBCO})$ coated conductors [1], which can now meet the requirements both for very high field magnets ( $>20 \mathrm{~T}$ at $4.2 \mathrm{~K})$ and large-scale power applications $(<3 \mathrm{~T}, 30-77 \mathrm{~K})$ [2]. Unfortunately, most devices in the latter category (including motors, generators and fault current limiters) are alternating current (AC) devices; and the high aspect ratio coated conductor geometry is associated with high losses in ac operation. The solution - to divide the REBCO film into narrower filaments, with transposition of those filaments along the length of the conductor-has long been known [3]; but implementing it on an industrial scale without increasing the cost per kA m, which is already prohibitive for many applications, has proved challenging.

Filament transposition can be achieved during cabling: for example, by winding around a cylindrical former (cable on round core, CORC) [4], or mechanically punching out narrower, shaped conductors and assembling them into a Roebel cable [5]. At the coated conductor fabrication stage, the emphasis is therefore on producing straight, in-plane REBCO filaments. Many methods have been proposed. The most common approach is to remove narrow strips of REBCO (and often the adjacent layers) by mechanical scribing, laser striation and/or etching processes [6]. Although effective and widely applicable, the removal of material is intrinsically wasteful, and inevitably reduces the current-carrying width of the conductor. These processes also operate on the brittle superconducting layer itself, the performance of which is very sensitive to defects, composition and microstructure.

The method proposed by Wulff et al [7, 8] also employs mechanical scribing and etching, but with two important innovations. The etching process is applied to the bare substrate before deposition of the REBCO film and accompanying buffer stack (using an adhesive film mask to pattern the filaments); and rather than minimising the width of the etched trenches, alternating etched and unetched tracks of equal width are prepared. The etching process is tuned to produce a small undercut, and Wulff describes the result as a 'two level undercut-profile substrate' (2LUPS). The buffer stack and REBCO film can then be deposited by conventional techniques. When line-of-sight methods such as PLD are used, the result is two parallel but vertically separated arrays of REBCO filaments, with potentially no reduction in total current-carrying width. The undercut regions are shadowed by the unetched top surface of the tape, reducing coupling between adjacent filaments. This architecture is not quite unique - a similar approach was patented for $\mathrm{Nb}_{3} \mathrm{Ge}$ in the 1970s [9] — but this is the first report of applying the concept to REBCO coated conductors.

The promising initial results reported here [7] confirm that the intended structure can be produced in a $150 \mathrm{~mm}$ long sample, and that, despite a very small undercut of only $0.3 \mu \mathrm{m}, 12$ distinct REBCO filaments can be formed in a $10 \mathrm{~mm}$ wide substrate. The deposition process was optimised for $4 \mathrm{~mm}$ wide tapes, so the critical current $\left(I_{\mathrm{c}}\right)$ cannot be expected to match the full-width coated conductor- 
depending on the number of filaments considered, the $I_{\mathrm{c}}$ reduction is $19-36 \%-$ but it is clear that REBCO filaments on both levels contributed to the total $I_{\mathrm{c}}$.

For comparison, recent results for $200 \mathrm{~mm}$ long laser-striated samples show an $I_{\mathrm{c}}$ decrease as low as $\sim 5 \%$ for a similar number of filaments [10]; whilst one of the few studies of long length striation reported a $20-50 \% I_{\mathrm{c}}$ degradation for $\sim 100 \mathrm{~m}$ long tapes with 3-5 filaments [6]. It therefore remains to be seen whether a comparable $I_{\mathrm{c}}$ to non-filamentary tape can be realised, and if this would be a compelling advantage over alternative methods.

Modifying the substrate prior to REBCO deposition introduces its own challenges. Non-planarity, roughness, and damage or residue from striation, could impede the growth of textured REBCO, especially inside the etched grooves. Similarly, local variations in substrate profile could result in interfilamentary coupling. For continuous filaments, the minimum local $I_{\mathrm{c}}$ dictates the overall current-carrying performance: so in long-length production, even rare and isolated defects are problematic. But it should be noted that the long-length reliability of alternative processes is similarly unproven.

In the present study, masking and scribing were performed manually; but the relative simplicity of the individual processes gives reason for optimism that this can be automated, and with lower equipment costs than laser striation. Wulff's patent application [8] also identifies other options for selective etching, and suggests that textured Ni-W substrates (RABiTS) and chemical solution deposition (CSD) are not excluded. The approach does, however, seem best suited to IBAD/ ABAD architectures and vapour deposition methods; and in that context, solution deposition planarisation (SDP) could potentially improve the surface quality of the etched tracks [11].

This approach is not without competition. Direct inkjet printing of REBCO filaments $[12,13]$ would more completely avoid materials wastage and reduce the number of processing steps. Inkjet printing also enables additive substrate modification routes, in which deposited tracks (rather than etched spacers) template or modify the growth of REBCO films [13, 14]. These approaches seem better suited than 2LUPS to CSD methods [15], which are not strictly line of sight. 'Inverse printing' methods could in principle also achieve full current-carrying width, by templating with thick textured filaments, and perhaps even allow three-dimensional filament transposition structures [13]; but this has yet to be proved experimentally.

Much remains to be demonstrated for the 2LUPS technique-not least a quantitative assessment of the hysteresis and coupling losses-and of course scaling to long lengths is the ultimate test; but this initial feasibility study is encouraging. If the authors can improve the surface quality, increase the undercut distance and fulfil the potential for matching the critical currents of full-width coated conductors, this interesting new approach could become the method of choice for filamentary coated conductor fabrication.

\section{Acknowledgments}

The author gratefully acknowledges funding from the European Union Seventh Framework Programme (FP7/2007-2013) project 'EUROTAPES' (grant agreement NMP-LA-2012-280432).

\section{References}

[1] Selvamanickam V, Heydari Gharahcheshmeh M, Xu A, Zhang Y and Galstyan E 2015 Critical current density above $15 \mathrm{MA} \mathrm{cm}^{-2}$ at $30 \mathrm{~K}, 3 \mathrm{~T}$ in $2.2 \mu \mathrm{m}$ thick heavily-doped $(\mathrm{Gd}, \mathrm{Y})$ $\mathrm{Ba}_{2} \mathrm{Cu}_{3} \mathrm{O}_{x}$ superconductor tapes Supercond. Sci. Technol. 28072002 
[2] Senatore C, Alessandrini M, Lucarelli A, Tediosi R, Uglietti D and Iwasa Y 2014 Progresses and challenges in the development of high-field solenoidal magnets based on RE123 coated conductors Supercond. Sci. Technol. 27103001

[3] Brandt E H and Indenbom M 1993 Type-II-superconductor strip with current in a perpendicular magnetic field Phys. Rev. B 4812893

[4] Šouc J, Gömöry F, Kováč J, Nast R, Jung A, Vojenčviak M, Grilli F and Goldacker W 2013 Low AC loss cable produced from transposed striated CC tapes Supercond. Sci. Technol. 26 075020

[5] Goldacker W, Grilli F, Pardo E, Kario A, Schlachter S I and Vojenčiak M 2014 Roebel cables from REBCO coated conductors: a one-century-old concept for the superconductivity of the future Supercond. Sci. Technol. 27093001

[6] Machi T, Nakao K, Kato T, Hirayama T and Tanabe K 2013 Reliable fabrication process for long-length multi-filamentary coated conductors by a laser scribing method for reduction of AC loss Supercond. Sci. Technol. 26105016

[7] Wulff A C et al 2015 Two level undercut-profile substrate for filamentary $\mathrm{YBa}_{2} \mathrm{Cu}_{3} \mathrm{O}_{7}$ coated conductors Supercond. Sci. Technol. 28072001

[8] Wulff A C 2013 Method for producing substrates for superconducting layers World Patent Application WO2013174380A1

[9] Marancik W G 1978 Composite multifilament superconductors US Patent Specification 4101731

[10] Nast R, Vojenčiak M, Demenčik E, Kario A, Ringsdorf B, Jung A, Runtsch B, Grilli F and Goldacker W 2014 Influence of laser striations on the properties of coated conductors J. Phys.: Conf. Ser. 507022023

[11] Sheehan C, Jung Y, Holesinger T, Feldmann D M, Edney C, Ihlefeld J F, Clem P G and Matias V 2011 Solution deposition planarization of long-length flexible substrates Appl. Phys. Lett. 98071907

[12] List FA, Kodenkandath T and Rupich M W 2007 Fabrication of Filamentary YBCO Coated Conductor by Inkjet Printing IEEE Trans Appl. Supercond. 17 3355-8

[13] Hopkins S C et al 2014 Inkjet printing of multifilamentary YBCO for low AC loss coated conductors J. Phys.: Conf. Ser. 507022010

[14] Cai X, Kesgin I, Schmidt R, Chen Y and Selvamanickam V 2013 Completely etch-free fabrication of multifilamentary coated conductor using inkjet printing and electrodeposition IEEE Trans. Appl. Supercond. 236603005

[15] Van Driessche I et al 2012 Chemical solution deposition using ink-jet printing for YBCO coated conductors Supercond. Sci. Technol. 25065017 